\title{
A review of the measurement of single crystal orientation parameters of nickel castings
}

\author{
$\mathrm{K}$ Clay $^{1, a}, \mathrm{P} N$ Quested $^{2, \mathrm{~b}}, \mathrm{R}$ Morrell $^{2}, \mathrm{~K}$ Mingard $^{2}$ \\ ${ }^{1}$ Hexmat Materials Consultancy Ltd, 24, Tor Rise, Matlock, Derbyshire, DE4 3DL, UK \\ ${ }^{2}$ Division of Materials, National Physical Laboratory, Teddington, Middlesex, TW11 OLW, UK \\ aclay@hexmat.u-net.com, b peter.quested@npl.co.uk
}

Key words: Nickel superalloys, Single crystal, Grain boundaries, Orientation measurement, Misorientation measurement, R-value, Round robin, Back reflection X-ray Laue pattern.

\begin{abstract}
Control of single crystal orientation in modern gas turbine components is a critical quality control issue for optimum reliable behaviour, and an increasingly important aspect of trade. This paper reviews industrial practice for the determination of primary crystal orientation and for the determination of 'disorientation' (R-value) between sub-grains. Different techniques and measurement conventions are compared, and the errors of measurement have been assessed through a European round-robin. Causes of poor reproducibility in 'disorientation' measurement have been identified by EBSD investigations. Recommendations for unified practice have been made in a Good Practice Guide as a precursor to a new industry-wide standard.
\end{abstract}

\section{Introduction}

Turbine industry and single crystal (SX) components. Since their introduction as high pressure turbine blades in jet engines in the 1980s, the exploitation of the property anisotropy afforded by SX nickel castings has expanded extensively. Their initial use provided: enhanced creep life, increased turbine entry temperatures, greater turbine efficiency and significant fuel savings. Subsequent uses include: lower pressure turbine blades, nozzle guide vanes (NGVs) and other structural turbine components in both aero and power generation turbines, where improved thermal fatigue control is achieved. In such high integrity applications, control of crystal orientation relative to key component features is both an engineering requirement and a manufacturing quality control parameter. The application-relevant properties of SX alloys are grain boundary sensitive. However, limitations in the directional casting process, especially for larger and more complex shaped components mean that a 'perfect' boundary free component is rarely produced. So OEMs have evolved component specific acceptance standards for allowable grain boundaries. These define limits for grain size and shape, grain boundary location and the misorientation between grains across a boundary, the R-value. The SX casting sector is growing 2008 's worldwide trade was circa $\$ 2.5$ billion, with a fifth in Europe [1]. During sector expansion, different definitions arose for the relevant parameters alongside variations in measurement methods. These are assessed and resulting standardisation proposals developed.

\section{Review of orientation parameter definitions.}

Main grain (primary) orientation. These comprise a systematic group of angles used to describe the orientation of the single crystal relative to a component-relevant reference system. This datum has a reference plane $\mathrm{RP}$, and reference direction $\mathrm{RD}$, lying within the $\mathrm{RP}$, Figure 1. Nickel superalloys have a face centred cubic (FCC) dominant crystal structure, so the primary orientation describes the orientation of the crystal $<001>$ direction closest to the component RD

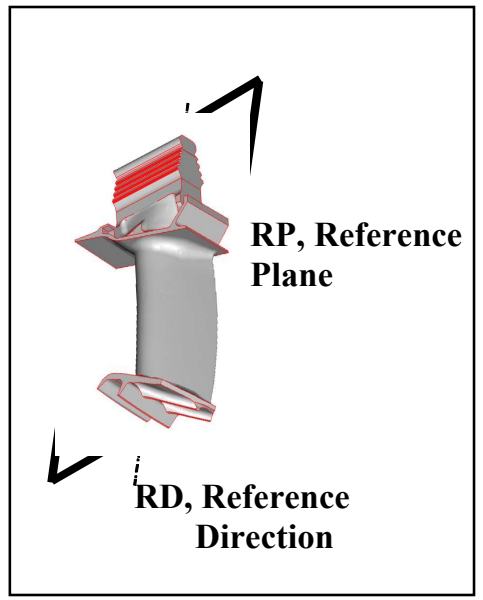

Figure 1. Component orientation references and the rotation of a crystal's $\{001\}$ plane parallel with the closest $<001>$ direction, from the component RP. 
Primary orientation angles. Primary orientation is measured by analysing Back Reflection Laue (BRL) film, as described in classical diffraction texts, [2, 3]. The wet film BRL method generates a pattern, shown schematically in Figure 2, where the pole of nearest $<001>$ direction, the zone representing a crystal's $\{001\}$ plane and the component references are all identified. A wet film is transparent so can be viewed from either side; these original texts assume that it is viewed looking towards the component, see Figure 3a. Some primary orientation angles can be measured directly on a pattern using a Greninger chart: they are gamma, $\gamma$, delta, $\delta$, and alpha, $\alpha$, also shown in Figure 2 where directions for positive rotations are indicated. This direction of viewing and definition convention is termed the European convention. Six angles are used in this convention as the additional parameters are of use to the OEMs. These extra angles are: theta, $\theta$, kappa, $\kappa$ and rho, $\rho$ : All can be calculated from the directly measured angles [4], Table 1. For full definitions see [5].

Primary orientation angles - alternate definitions. When the same crystal alignment film is viewed from the opposite side, the observed position of the diffraction pattern is different, Figure 3. To obtain the same values for directly measured primary orientation angles, adjustments will need to be made to their sense (direction of rotations). In the US convention, the BRL pattern (hence component) is viewed looking back at the film i.e. towards the X-ray source (Figure $3 b$ ). However, in this convention, the definitions of positive sense are adjusted for only two directly measured angles (designated gamma and delta), but the definition of positive rotation direction of the third angle (designated beta,), is unadjusted. So, comparing their positive rotations when viewing a component's examined surface: gamma and delta have equivalent positive directions,

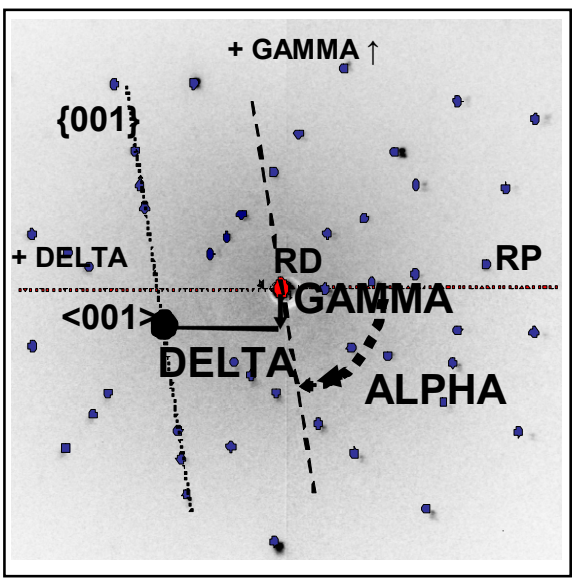

Figure 2. Annotated Back Reflection Laue Image (a) Direction of view, wet film

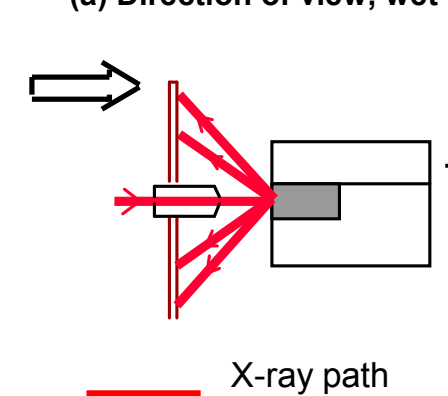

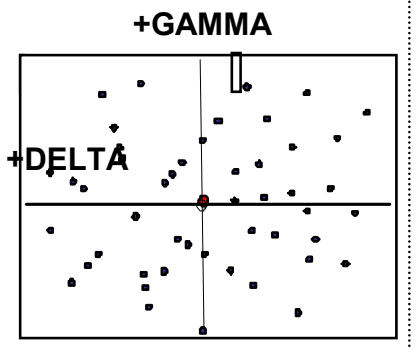

Figure 3. and thus the same numerical values, but European alpha and US beta have opposite positive sense, and so different numerical values. The main differences between the conventions are shown in Table 2. A second, less common, US2 convention exists with a different approach for beta, see [5]. All three conventions are used in the SX casting industry, and this can (b) Direction of view, Polaroid film

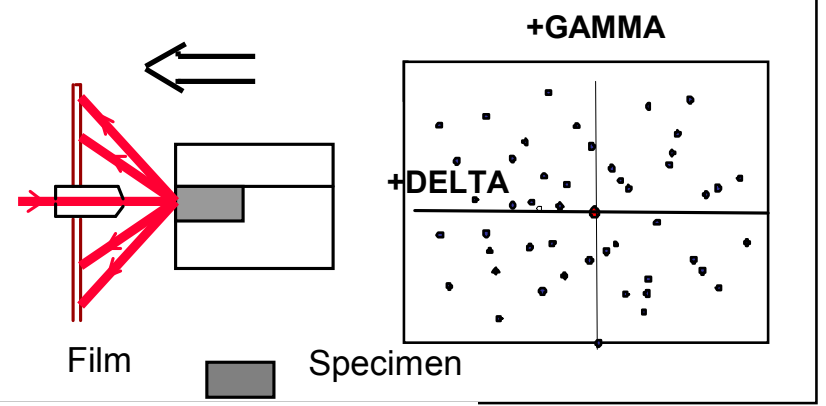

\section{BRL diffraction film viewing options}

Table 1. Calculation of derived primary angles from directly measured angles - European convention.

\begin{tabular}{|c|c|c|}
\hline Derived angle & Formula & Notes: \\
\hline theta $(\theta)$ & $\cos \theta=\cos \gamma \cdot \cos \delta$ & \\
\hline kappa (к) & $\kappa=\kappa_{\mathrm{TRUE}}-\operatorname{atan}(\tan \gamma \cdot \sin \delta)$ & $\begin{array}{l}* \text { See below for } \\
\text { definition } \kappa_{\text {TRUE }}\end{array}$ \\
\hline rho $(\rho)$ & $\rho=\operatorname{atan}(\tan \gamma / \sin \delta))-\kappa_{\mathrm{TRUE}}$ & $\begin{array}{l}* \text { See below for } \\
\text { definition } \kappa_{\mathrm{TRUE}}\end{array}$ \\
\hline $\begin{array}{c}* \text { kappa TRUE } \\
\left(\kappa_{\text {TRUE }}\right)\end{array}$ & $\begin{aligned} \tan \left(\kappa_{\mathrm{TRUE}}\right)= & \cos \gamma \cdot \tan \alpha /(\cos \delta-\sin \gamma . \\
& \sin \delta \cdot \tan \alpha)\end{aligned}$ & \\
\hline
\end{tabular}


Table 2. Comparison of European and US Primary orientation conventions.

\begin{tabular}{|c|c|c|}
\hline Attribute & $\begin{array}{c}\text { EUROPEAN } \\
\text { convention }\end{array}$ & $\begin{array}{l}\text { US convention } \\
\text { (US1) }\end{array}$ \\
\hline VIEWING & VIEWING THE & VIEWING THE X- \\
\hline CONVENTION & COMPONENT. & RAY SOURCE. \\
\hline SENSE OF & CLASSICAL & CLASSICAL \\
\hline ANGLES & DEFINITION. & DEFINITION. \\
\hline ANGLE & GAMMA, $\gamma^{1}$ & GAMMA, $\gamma^{1}$ \\
\hline NAMES: & DELTA, $\delta$ & DELTA, $\delta$ \\
\hline $\begin{array}{r}{ }^{1}=\text { equivalent } \\
\text { measureme }\end{array}$ & THETA, $\theta^{1}$ & ALPHA, $\alpha^{1}$ \\
\hline $\begin{array}{l}\text { nt and } \\
\text { value. }\end{array}$ & ALPHA, $\alpha^{2}$ & BETA, $\beta^{2}$ \\
\hline${ }^{2}=$ equivalent & КАРРА, к & - \\
\hline $\begin{array}{l}\text { measuremen, } \\
\text { different } \\
\text { value. }\end{array}$ & RHO, $\rho$ & \\
\hline
\end{tabular}

European uses 6 angles, US1 convention uses 4 angles lead to confusion for users and suppliers of primary orientation data. One needs to explicitly define and know the convention applied especially if comparing components and materials or defining process controls.

\section{Grain boundary misorientation R-values.}

The R-value, a number in units of angle $\left(^{\circ}\right)$, is used to assess grain 'misorientation' or, more correctly, 'disorientation', as it is the minimum angular displacement between adjacent grains. The R-value can be directly correlated with the behaviour of a SX component in service. Industry acceptance limits, which are component specific, and even sometimes zone specific within a component, are in the region of $8^{\circ}$. These may increase to $10-12^{\circ}$ or even $18^{\circ}$ if grain stabilised alloys are introduced. If grains on either side of a boundary are designated A and $\mathrm{B}$, the R-value represents a summary of the rotations required to align grain $\mathrm{A}$ with grain $\mathrm{B}$. This requires orientations of each grain to be measured relative to the same arbitrary reference system, which does not need to be defined relative to the component shape since boundaries can occur at any location on its surface, see [3]

and [5] for a full description of misorientation definitions. Within the SX industry, various R-value definitions have evolved. Three general categories exist: single-angle, two-angle (REL) and difference (three-angle) methods. All are detailed in Table 3. For a more rigorous mathematical treatment of orientations and rotations see [4, 5 and 6$]$. Note that different R-value conventions can produce different values for the same physical grain boundary. Theoretical calculations [7] show a consistent correlation of the single-angle (R-1cos) and two-angle (R-2cos) methods as they track together up to $60^{\circ}$, whereas the three angle, R3RMS, method diverges significantly from the others in some regions of the $\mathrm{R}$-value range. This is supported by comparing results from various industrial facilities using the different conventions, Table 4.

Table 3. R-value definition methods used in the SX industry.

\begin{tabular}{cc}
\hline Name & Formula \\
\hline $\begin{array}{c}\text { Single-angle } \\
\text { cos, (R-1cos) }\end{array}$ & $R=$ Minimum of 24 single rotations \\
Two-angle cos, & \\
(REL or R- & \\
2cos) & $R=\cos ^{-1}(\cos \phi \cos \tau)$ \\
Two-angle & $R=\sqrt{\left(\phi^{2}+\tau^{2}\right)}$ \\
RMS, (R- & \\
2RMS) & \\
Three-angle & $R=\cos ^{-1}\left(\cos \left(\gamma_{A}-\gamma_{B}\right) \cos \left(\delta_{A}-\delta_{B}\right) \cos \left(\alpha_{A}-\alpha_{B}\right)\right)$ \\
cos, (R-3COS) & \\
Three-angle & $\left.R=\sqrt{\left(\left(\gamma_{A}-\gamma_{B}\right)^{2}+\left(\delta_{A}-\delta_{B}\right)^{2}+\left(\alpha_{A}-\alpha_{B}\right)^{2}\right.}\right)$ \\
RMS, (R- & $R=$ \\
3RMS) & \\
\hline
\end{tabular}

Where $\gamma, \delta, \alpha$ are the orientation angles, European convention, as defined in [4] for boundary grains $\mathrm{A}$ and $\mathrm{B}$ respectively (but can be replaced by

$\gamma, \delta, \beta$ respectively, US convention) and $\phi, \tau$ are the misorientation angles as defined in [5]. 
Table 4. Examples of as-received average $\mathrm{R}$-values in a round robin exercise.

\begin{tabular}{|c|c|c|c|c|}
\hline \multirow{2}{*}{ Specimen } & \multicolumn{4}{|c|}{$\begin{array}{l}\text { R-values determined using different calculation } \\
\text { methods at different facilities }(\mathrm{W}, \mathrm{X}, \mathrm{Y}, \mathrm{Z})\end{array}$} \\
\hline & $\mathbf{W}$ & $\mathbf{X}$ & $\mathbf{Y}$ & $\mathbf{Z}$ \\
\hline $\operatorname{Ra}\left[^{\circ}\right]$ & 7.0 & 7.3 & 7.5 & 6.8 \\
\hline $\mathbf{R b}\left[{ }^{\circ}\right]$ & 10.8 & 11.7 & 11.1 & 10.8 \\
\hline $\operatorname{Rc}\left[^{\circ}\right]$ & 12.7 & - & 16.4 & 12.8 \\
\hline Rd [ $\left.{ }^{\circ}\right]$ & 32.8 & 41.0 & 44.4 & 34.3 \\
\hline $\operatorname{Re}\left[{ }^{\circ}\right]$ & 52.4 & 78.9 & 51.2 & 50.9 \\
\hline
\end{tabular}

To minimise the impact of these variants, it is recommended that only the $\mathrm{R}-1$ cos (singleangle) and R-2cos (two-angle) methods are used. Even though $\mathrm{R}-1 \cos$ alone is mathematically rigorous over the complete numerical range for disorientation (0 to $\left.62.8^{\circ}\right)$, $\mathrm{R}-2 \cos$ is also recognised as it is widely used and embedded within design criteria.

\section{Review of orientation measurement methods.}

Production measurement uses systems based on the X-ray diffraction BRL technique, shown in

Figure 3. It is a surface-only measurement, (penetration depth of 2-5 $\mu \mathrm{m}$ and a 1.5-2 mm diameter analysed area) so it has an associated assumption that the measurement point represents the whole casting and also a requirement that this surface layer must be strain / damage free. The film registers diffraction poles (spots) that form a pattern having the FCC system's symmetry characteristics and a position determined by the alignment of the crystal relative to the measurement references. The logical phases of BRL pattern analysis are: (i) unambiguous identification of pattern symmetry, (ii) definition of the position of the pattern (location and rotation) and (iii) use of these data to derive the primary orientation angles. System alignment and set up constraints are detailed in [5]. The elapsed time for obtaining orientation from a wet film can be up to 1 hour. Thus to improve production inspection efficiency, 3 categories of system feature variants have been introduced, all exploit technology advances from other fields.

Image capture methods. First, instant "Polaroid" film replaced wet film. Such films can only be viewed in one direction i.e. looking towards the X-ray source, so require appropriate adjustments to diffraction pattern analysis to attain equivalent orientation angles. The introduction of real-time Laue digital imaging reduced image collection times to 10 seconds or less. But, these bespoke area detectors require major changes to the BRL system geometry as the X-ray beam cannot be directed through them.

System geometry. Adaptations of the true BRL geometry resulted in side reflection systems. As well as accommodating real-time area detectors, these changes also increase the space available for larger components. The changes are systematic and can be described mathematically, so orientation measurements equivalent to those obtained in a true BRL geometry are feasible. In side reflection, a system parameter, $\mathrm{S}$ (the working distance), is a key variable that must be precisely known. So, independent system scaling routines (using silicon single crystal standards) evolved and are now essential for all production Laue measurement systems. [5]

Pattern analysis protocol. Pattern analysis protocols fall into three groups. The previously outlined charting methods, have typical analysis times of over 15 minutes and require the analyst to have a detailed understanding of crystal symmetry; both are non-ideal attributes for production measurements. The second group, full mask pattern matching, uses computer generated overlay masks comensurate with the measurement system geometry. These are either the more common full spot pattern format or, a line mask: the latter however, can be subject to systematic matching, hence measurement errors. The operator selects and matches a mask with the diffraction pattern image. The total cycle time for a pattern matching routine is between 30-60 seconds. Finally, the marking of individual diffraction spots co-ordinates within a calibrated field. These are commonly used with films and require data entry in specified groups, e.g. along a diffraction zone line, to simplify pattern symmetry identification. Cycle times are 1-2 minutes. 


\section{Experimental Assessments}

Laue X-ray systems. A round robin measurement review was completed for both primary and Rvalues. For each, 8 production pieces were used: comprising a range of alloy / material condition combinations and covering the total range value for each parameter, thus representative of the industry's components, see Table 5 for measurement details. Statistical assessments were completed conforming to ASTM E 691-05 (inter-laboratory measurement studies) [8].

EBSD (Electron Back Scattered Diffraction). Three R-value samples, previously examined by BRL, were prepared for EBSD. Acquisition and data processing settings for EBSD were chosen to achieve good angular accuracy. Orientations were measured at $10 \mu \mathrm{m}$ intervals across a strip up to $7 \mathrm{~mm}$ either side of the boundary; field map stitching data combination was used.

\section{Results, discussions and measurement implications.}

A summary of the analysis of the round robin review is shown in Table 5. Overall parameter measurement uncertainties (using reproducibility standard deviations, $\sigma_{\mathrm{R}}$, at the $95 \%$ confidence

Table 5. Statistical summary of round robin assessment of X-ray Laue measurements. 5 facilities, 16 specimens ( 8 primaries, P: 8 R-values, R), 5 repeat measurements of each at every facility: all primaries European convention, all R-Values calculated by R-2cos method: statistical methods as defined in [8]. All values [ ${ }^{\circ}$.

\begin{tabular}{|c|c|c|c|c|c|c|c|c|c|}
\hline Specimen & P1 & $\mathbf{P 2}$ & \multicolumn{2}{|c|}{ P8 } & P13 & $\mathbf{P 2 7}$ & \multicolumn{2}{|c|}{ P11 } & P21 \\
\hline Theta / kappa & $1.9 / 9.0$ & $8.1 / 6.9$ & \multicolumn{2}{|c|}{$14.5 / 79.88$} & $20.1 / 52.8$ & $27.1 / 56.1$ & \multicolumn{2}{|c|}{$37.6 / 19.1$} & $41.4 / 59.3$ \\
\hline s.d. & $0.28 / 0.62$ & $0.39 / 0.88$ & \multicolumn{2}{|c|}{$0.25 / 0.92$} & $0.34 / 0.78$ & $0.39 / 0.7$ & \multicolumn{2}{|c|}{$0.26 / 0.86$} & $0.13 / 0.68$ \\
\hline$\sigma_{\mathrm{r}}$ & $0.23 / 0.36$ & $0.19 / 0.46$ & \multicolumn{2}{|c|}{$0.11 / 0.35$} & $0.17 / 0.41$ & $0.09 / 0.38$ & \multicolumn{2}{|c|}{$0.2 / 0.32$} & $0.21 / 0.3$ \\
\hline$\sigma_{\mathrm{R}}$ & $0.34 / 0.7$ & $0.39 / 0.97$ & \multicolumn{2}{|c|}{$0.27 / 0.98$} & $0.37 / 0.86$ & $0.4 / 0.78$ & \multicolumn{2}{|c|}{$0.31 / 0.91$} & $0.23 / 0.73$ \\
\hline \multicolumn{2}{|c|}{ Specimen } & R1 & R3 & R5 & R6 & R9 & R11 & R13 & R14 \\
\hline \multicolumn{2}{|c|}{ R-value } & 3.3 & 7.1 & 10.7 & 12.7 & 22.9 & 43.9 & 33.4 & 49.8 \\
\hline \multicolumn{2}{|c|}{ Standard deviation (s.d) } & 0.87 & 0.09 & 0.24 & 0.41 & 0.23 & 1.75 & 0.59 & 2.05 \\
\hline \multicolumn{2}{|c|}{ Repeatability, $\sigma_{\mathrm{r}}$} & 1.16 & 0.38 & 0.52 & 0.69 & 0.33 & 0.42 & 0.44 & 0.47 \\
\hline \multicolumn{2}{|c|}{ Reproducibility, $\sigma_{R}$} & 1.36 & 0.3 & 0.53 & 0.74 & 0.37 & 1.79 & 0.71 & 2.10 \\
\hline \multicolumn{10}{|c|}{ One primary specimen omitted } \\
\hline
\end{tabular}

level [8]) were established as $\pm 0.8^{\circ}, \pm 2.0^{\circ}$ and $\pm 1.5^{\circ}$ for theta, kappa and R-value respectively. On comparison with the limits of acceptability for measurement precision demanded by OEMs (respectively, $\pm 1.0^{\circ}, \pm 1.0^{\circ}$ and $\pm 1.5^{\circ}$ ), only the uncertainty with kappa appeared outside expectations. Review of the detail of measurement methods indicated that the kappa (and alpha) assessments are susceptible to system misalignment, highlighting the importance of rigorous system alignment and calibration monitoring. For R-values, individual $\sigma_{R}$ values varied widely, $0.37-2.1^{\circ}$, and it was noted that such uncertainties were generally dominated by the repeatability for each facility. These larger $\sigma_{R}$ values were often associated with a potential freedom to vary the positions selected for the orientation measurements of both boundary grains. Ideally, these measurements should be at the same position with the X-ray beam straddling

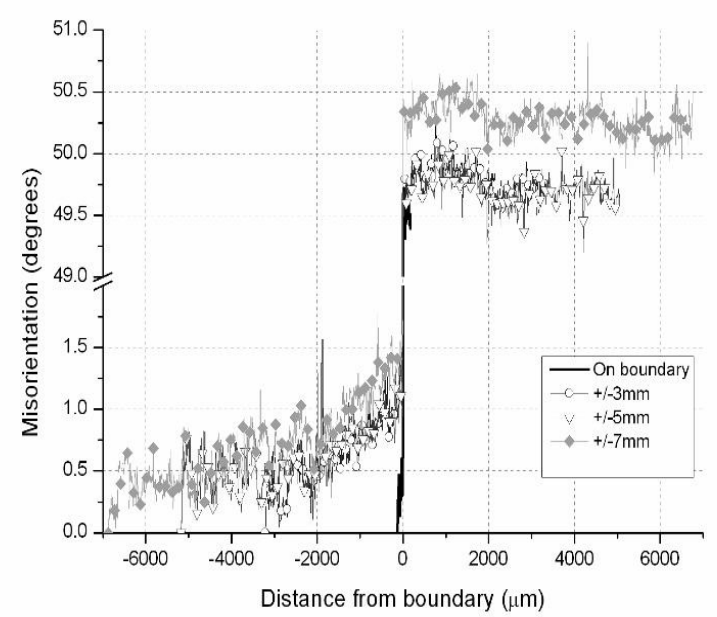

Figure 4. EBSD Changes in orientation across a grain boundary, specimen R14 (SEM:Zeiss Supra 400, FEG, 20kV) 
the boundary (a one shot method). But some practitioners measure each grain at distances up to $15 \mathrm{~mm}$ from the boundary. Further repeat R-value assessments were completed for specimen R14 (R $=49.8^{\circ}$ ) at known measurement positions. This extended data set raised $\sigma_{\mathrm{R}}$ from 2.1 to 2.24 . Also the two assessments made directly on the boundary itself generated different R-values, proven as statistically significant by d-statistic evidence [8]. A microstructural explanation for this apparent Rvalue measurement position dependency was the purpose of the EBSD investigation. Figure 4 plots the misorientation angle for specimen R14 between the starting point and each subsequent point along a line drawn across the grain boundary. EBSD determines misorientation as an angle/axis pair, but only the angle is shown. A significant variation in misorientation with the distance from the boundary is shown. Other samples examined showed either different orientation change trends or constant orientation when approaching a boundary. Where changes were observed, they were shown to be consistent with complimentary twists in grains on either side of the boundary, a probable boundary strain accommodating mechanism. This study provides an explanation for the observed Laue R-value measurement position dependency. A recommendation for improving overall R-value measurement uncertainty follows: ensure that both grain measurements are made directly on the boundary with the X-ray beam straddling the interface.

\section{Implications of reviews.}

The work and findings reported were part of an 18 month European industry collaborative programme examining nickel casting orientation assessment and its fitness for purpose. The project resulted in a Good Practice Guide [5]. The publication addresses issues relating to the reliability and precision of the orientation measurements made in different establishments and covers: definition of terms and angles, good practice in set up and calibration of X-ray systems, identification of X-ray patterns and their measurement, differences between European and US conventions for angles and assessment of uncertainties of measurement, supported by an inter-laboratory exercise. The document is a proposed precursor for an industry-wide standard.

\section{Acknowledgements.}

The authors wish to thank several Industrial Companies for technical and financial support and also recognise financial support from the UK's National Measurement Office.

\section{References}

[1] D. Ford, European Investment Casting Federation, - private communication, 2010.

[2] B.D. Cullity: Chapters 3 and 8 in Elements of X-Ray Diffraction, Second Edition. Addison Wesley, London, 1978, ISBN 0-201-01174-3.

[3] ASTM E82-91 (Re-approved 2007): Standard Test Method for Determining the Orientation of a Metal Single Crystal, ASTM International, 2007.

[4] L.N. McCartney and K. Clay: Rigorous Analysis for Estimating the Orientation of Cubic Crystals, NPL Report MAT 30, 2009, National Physical Laboratory, Teddington, UK

[5] K. Clay, J.D. Jackson, P.N. Quested and R Morrell: Improving Single Crystal Orientation Determination for Advanced Nickel-Based Superalloys. Measurement Good Practice Guide No. 112, Issue 1, April 2009. National Physical Laboratory, Teddington, UK. ISSN 1368-6550. http://publications.npl.co.uk/dbtw-wpd/exec/dbtwpub.dll

[6] V. Randle, and O. Engler: Introduction to Texture Analysis Macrotexture, Microtexture and Orientation Mapping, CRC Press, Boca Raton, USA, 2000, ISBN 90-5699-244-4.

[7] Information on http:// www.jacksonelectronics.co.uk, accessed April 2008

[8] ASTM E691-05: Standard Practice for Conducting an Interlaboratory Study to Determine the Precision of a Test Method. ASTM International, 2005. 